\section{Urinary symptoms and urody- namic findings before and after vaginal surgery for pelvic organ prolapse}

Narisra Srikureja, Pattaya Hengrasmee, Pichai Leerasiri, Chutimon Asumpinwong

Urogynecology Unit, Department of Obstetrics and Gynecology, Faculty of Medicine, Siriraj Hospital, Mahidol University, Bangkok, Thailand

\begin{abstract}
To ascertain the difference in urodynamic findings, specifically bladder sensation, and urinary symptoms after vaginal surgery for Pelvic Organ Prolapse (POP). Retrospective data analysis of 126 women who underwent vaginal surgery for POP without simultaneous anti-incontinence procedure from January 2013 to April 2019 at Siriraj Hospital, Thailand. Baseline characteristics, intraoperative details and pre and post-operative urinary symptoms and urodynamic findings were recorded. There was no significant difference in the pre and post-operative first desire to void, at $158 \pm 53 \mathrm{~mL}$ and $162 \pm 64 \mathrm{~mL}$, respectively $(\mathrm{p}=0.518)$. Incidence of increased bladder sensation was also unchanged, from $46.0 \%$ to $46.8 \%(\mathrm{p}=1.00)$. Post-operative urodynamic stress incontinence was significantly increased, from $15.9 \%$ to $31.0 \%(\mathrm{p}=0.003)$, as was the incidence of weak bladder contractility index $(<100)$, from $47.3 \%$ to $61.8 \%(p=0.005)$. Significant improvements in post-operative urge urinary incontinence, urgency and voiding dysfunction were noted, from $50.8 \%$ to $31.7 \%$ $(\mathrm{p}=0.001), 63.5 \%$ to $38.9 \%(\mathrm{p}<0.001)$ and $42.9 \%$ to $5.6 \%(p<0.001)$, respectively. No significant difference in bladder sensation after vaginal surgery for POP repair was noted. However, urinary symptoms significantly improved after surgery.
\end{abstract}

\section{Introduction}

Pelvic Organ Prolapse (POP) is a condition where the pelvic organs and the overlying vaginal segments protrude into the vagina or through vaginal orifice. ${ }^{1}$ Due to their anatomical association, POP and urinary incontinence have similar risk factors and are often found in conjunction with each other. ${ }^{2}$ Moreover, Lower Urinary Tract Symptoms (LUTS) such as frequency, urgency, Urgency Urinary Incontinence
(UUI), Stress Urinary Incontinence (SUI) and voiding dysfunction are often prevalent in patients with pelvic organ prolapse. ${ }^{3}$ There are a variety of surgical procedures available for POP repair, for which is suitable, depending on the location and the severity of prolapse, associated symptoms ${ }^{4}$ and the surgeon's preferred route of operation. However, $80-90 \%$ of the procedures are undertaken via the vaginal route..$^{5,6}$ Correction of POP can either ameliorate or exacerbate these LUTS. Previous studies focusing on urinary symptoms found a 6$22 \%$ incidence of de novo SUI after prolapse repair. ${ }^{7-10}$ It is suggested that prolapse of pelvic organs can cause urethral kinking, and POP repair restores the normal urethral anatomy unmasking the symptom of SUI that may have been covertly present before the operation. ${ }^{7,11-13}$ Also, extensive pelvic reconstructive surgery can interfere the lower urinary tract nerve plexuses resulting in the development of de novo SUI. ${ }^{14}$ Some studies additionally demonstrated an increase in de novo UUI, ${ }^{7,8}$ whilst others showed improvement postoperatively. ${ }^{15-17}$ Lastly, voiding dysfunction which often presents with POP tends to improve after POP repair. ${ }^{3,17}$

Apart from changes in LUTS, several previous literatures comparing pre- and post-operative urodynamic findings in patients undergoing pelvic floor reconstruction for prolapse consistently demonstrated improvement in voiding phase parameters after surgery, such as higher maximum urine flow rate $\left(\mathrm{Q}_{\max }\right)$, reduced Postvoid Residual Urine (PVR), and disappearance of Bladder Outflow Obstruction (BOO), ,16${ }^{17}$ which resulted from resolution of urethral kinking. However, conflicting data have still persisted when evaluating in terms of filling phase parameters, including bladder sensation. Previous studies assessing urodynamic changes after laparoscopic sacrocolpopexy reported inconsistent results. While Illiano et al. ${ }^{16}$ found no significant differences in cystometric capacity pre- and post-operatively, Abdullah et al. ${ }^{17}$ and Kummeling et al. ${ }^{18}$ showed remarkable improvement in first desire volume, strong desire volume and bladder capacity. Furthermore, when looking at a comparative urodynamic analysis in patients undergoing vaginal operations for POP, Panicker et al. ${ }^{3}$ failed to demonstrate any difference in bladder sensation before and after surgery.

Therefore, due to these contradictory results, our study primarily aimed to ascertain the differences in pre- and post-operative urodynamic filling phase parameters in terms of bladder sensation among patients undergoing vaginal surgery for POP repair.
Correspondence: Chutimon Asumpinwong, Urogynecology Unit, Department of Obstetrics and Gynecology, Faculty of Medicine, Siriraj Hospital, Mahidol University, Bangkok, 2 Wanglang Road, Bangkok 10700, Thailand.

Tel.: +66.2419.4782; Fax: +66-2419-4783

E mail: chutata@hotmail.com

Key words: Pelvic organ prolapse; vaginal surgery; lower urinary tract symptoms; urodynamic findings.

Contributions: NS: protocol development, data collection, data analysis, manuscript writing; PH: data analysis, manuscript editing; PL: protocol development; CA: protocol development, data analysis, manuscript editing.

Conflict of interest: The authors have no conflict of interest to declare.

Conference presentation: This research work was presented in an oral format at the annual scientific meeting of the Royal Thai College of Obstetricians and Gynaecologists on 17th October, 2019.

Availability of data and materials: All data generated or analyzed during this study are included in this published article.

Ethics approval and consent to participate: This study was approved by the ethics committee of the siriraj institutional review board [approval code 520/2561 (ec1)]. This study conformed with the helsinki declaration of 1964, as revised in 2013, concerning human and animal rights.

Informed consent: Since the study design was a retrospective chart review, the IRB did not require patient's informed consent.

Received for publication: 27 November 2020. Revision received: 30 April 2021.

Accepted for publication: 15 June 2021.

This work is licensed under a Creative Commons Attribution NonCommercial 4.0 License (CC BY-NC 4.0).

(C) Copyright: the Author(s), 2021

Licensee PAGEPress, Italy

Urogynaecologia 2021; 33:264

doi:10.4081/uij.2021.264

Secondarily, we planned to investigate changes in both LUTS and other urodynamic parameters.

\section{Materials and Methods}

After approval from the Ethics Committees of Siriraj Institutional Review 
Board (protocol number 520/2561 EC1), the medical records of women diagnosed with at least stage II uterovaginal prolapse undergoing transvaginal POP repair at the Urogynecology Unit, Department of Obstetrics and Gynaecology, Siriraj Hospital, Bangkok, Thailand during January 2013 and April 2019 were retrospectively reviewed. To minimize confounding factors, we recruited only those who had undergone vaginal hysterectomy with additional transvaginal repair(s), except anti-incontinence procedure. These adds-on transvaginal repairs included anterior and/or posterior colporrhaphy, anterior vaginal mesh repair, apical suspension (uterosacral ligament suspension or sacrospinous fixation), and obliterative total colpocleisis. All the women included in our study underwent urodynamic assessment before, and 6 months after the operation. Patients who had prior anti-incontinence surgery were excluded from this study.

The data collection comprised patients' baseline characteristics (age, parity, BMI, menopausal status, and hormone use), preand post-operative POP stage and location, pre- and post-operative LUTS, perioperative outcomes, as well as pre- and postoperative urodynamic findings.

Clinical assessment for the stage and the location of POP was carried out in accordance with the Pelvic Organ Prolapse Quantification (POP-Q) system. ${ }^{1}$ In addition, POP-related LUTS were evaluated using symptom questionnaire and a 3-day frequency/volume chart. According to the International Urogynecological Association (IUGA)/International Continence Society (ICS) joint report on the terminology for female pelvic floor dysfunction, ${ }^{19}$ LUTS are categorized into urinary incontinence, bladder storage, sensory, and voiding symptoms. For urinary incontinence symptoms, "stress urinary incontinence" is defined as a complaint of involuntary loss of urine on effort or physical exertion, "urgency urinary incontinence" is described as a complaint of involuntary loss of urine associated with urgency, and "mixed urinary incontinence" represents a complaint of involuntary loss of urine associated with both urgency and with effort or physical exertion. For bladder storage symptoms, our study mainly focused on "urgency" which is a complaint of a sudden and compelling desire to pass urine that is difficult to defer. Bladder sensation can be classified as "increased" (desire to void during bladder filling occurs earlier or is more persistent), "reduced" (desire to void occurs later to that previously experienced despite an awareness that the bladder is filling), or "absent" (absence of the sensation of bladder filling and a defi- nite desire to void). Lastly, all abnormal symptoms reported during or following micturition, including slow stream, intermittent flow, incomplete bladder emptying, and straining to void, are determined as "voiding symptoms". Impact of POP and POP-related urinary symptoms on patients' quality of life was simply assessed using a four-point scoring scale ranging from 0 ("none"), through 1 ("mild") and 2 ("moderate") to 3 ("severe").

After thorough evaluation, women who were diagnosed with at least stage II symptomatic POP and had failed or denied conservative treatments for POP were offered surgical correction. Vaginal hysterectomy was indicated among those with concurrent benign uterine pathology (e.g., leiomyoma, adenomyosis, endometrial polyp, etc.) or on patients' request due to their perception of the prolapsed uterus as an abnormality. Urodynamic study was scheduled for every individual prior to and 6 months after surgery irrespective of any LUTS experienced. POP beyond hymen was reduced with vaginal pessary during urodynamic study in order to unmask any occult SUI.

A complete ICS-standard urodynamic test that comprised i) free uroflowmetry and PVR and ii) cystometry and pressure-flow study was conducted. ${ }^{20}$ Initially, free uroflowmetry was performed after achieving bladder fullness to investigate maximum urine flow rate $\left(\mathrm{Q}_{\max }\right)$ and voided volume. Postvoid Residual Urine (PVR) was later measured by catheterization. After zero setting, filling cystometry was undertaken by inserting fluid filled catheters, with external transducers, into the bladder for normal saline instillation $(50 \mathrm{~mL} / \mathrm{min})$ and for intravesical pressure $\left(\mathrm{P}_{\mathrm{ves}}\right)$ determination. Another catheter was inserted into the rectum to measure abdominal pressure $\left(\mathrm{P}_{\mathrm{abd}}\right)$ and calculate for detrusor pressure $\left(\mathrm{P}_{\mathrm{det}}\right)$. While the bladder was gradually filled up, any noticeable involuntary detrusor contractions (Detrusor Overactivity; DO), cough-induced urine leakage, and the three sensation parameters, including First Desire to Void (FDV), Strong Desire to Void (SDV), and urgency were recorded. According to the definition by IUGA/ICS joint report on the terminology for female pelvic floor dysfunction, ${ }^{19} \mathrm{FDV}$ is the first feeling that the woman may wish to pass urine, SDV is the persistent desire to pass urine without the fear of leakage, and urgency is the compelling desire to pass urine which is difficult to defer. A female bladder usually experiences a first desire to void at a volume of approximately 150 to $250 \mathrm{~mL}$, a normal desire to void at 300 to $400 \mathrm{~mL}$, and a strong desire to void at 400 to $600 \mathrm{~mL}$ Therefore, we defined "reduced" bladder sensation as a FDV exceeding 250 $\mathrm{mL}$ and "increased" bladder sensation as a FDV of less than $150 \mathrm{~mL}$. Bladder compliance, defined as the relationship between a change in bladder volume and a change in detrusor pressure $\left(\Delta \mathrm{V} / \Delta \mathrm{Pdet} ; \mathrm{mL} / \mathrm{cmH}_{2} \mathrm{O}\right)$, was also assessed. In non-neurogenic bladders, the value over $40 \mathrm{~mL} / \mathrm{cmH}_{2} \mathrm{O}$ is considered as normal bladder compliance. ${ }^{21}$ Bladder filling was stopped after reaching urgency or $500 \mathrm{ml}$ of instillation. Provocative stress test (cough and Valsalva) was then performed to confirm the presence or absence of Urodynamic Stress Incontinence (USI) and cough-induced DO. Hand washing was another strategy implemented to detect DO.

Pressure-flow study started after permission to void during which maximum urine flow rate $\left(\mathrm{Q}_{\max }\right)$, detrusor pressure at maximum flow $\left(\mathrm{P}_{\text {det }} \mathrm{Q}_{\max }\right)$, and PVR were obtained. With regards to Blaivas-Groutz criteria, ${ }^{22}$ bladder outflow obstruction (BOO) was diagnosed. For bladder contractility, this was categorized according to Schafer's Bladder Contractility Index (BCI). Using their formula $\left(B C I=P_{\text {det }} Q_{\max }\right.$ $\left.+5 \mathrm{Q}_{\max }\right)$, strong bladder contractility was defined as an index value of more than 150 , whereas weak contractility was indicated by the BCI less than $100 .{ }^{23}$

Three urogynecologists were responsible for all surgical procedures. Vaginal hysterectomy was undertaken using a standard technique as practiced by all gynecologists at our center. After having been anesthetized, the patient was placed in lithotomy position and an indwelling catheter was inserted for bladder drainage. The cervix was grasped with tenaculum, followed by submucosal injection of saline or sterile water to separate the vaginal fascial layers. Following circumferential incision around vaginal fornix, blunt dissection into vesicovaginal and rectovaginal spaces was achieved to mobilize the bladder and the rectum away from the surgical field. Both uterosacral and cardinal ligaments were then clamped, cut, and suture-ligated with 1-Vicryl. Peritoneal cavity was entered either via posterior cul-de-sac or vesicouterine pouch. After cutting and securing both uterine vessels, broad ligaments and adnexal pedicles were also clamped, cut, and suture-ligated with 1-Vicryl. Vaginal cuff closure was performed after checking and securing all pedicles for hemostasis. Having completed vaginal hysterectomy, additional pelvic floor reconstructive procedures including anterior colporrhaphy, posterior colporrhaphy, anterior vaginal mesh repair, apical suspension (uterosacral ligament suspension or sacrospinous fixation), and/or obliterative total colpocleisis, were carried 
out as needed.

Postoperative follow-up was scheduled at 6 weeks, 6 months, and annually after surgery. At each visit, re-evaluation of POP stage and location as well as postoperative LUTS was performed. Urodynamic study was repeated at 6-month postoperative visit as previously mentioned. At 6 months follow-up, POP recurrence was diagnosed according to the NICHD (National Institute of Child Health and Human Development) Pelvic Floor Disorders Network recommendations $^{24}$ as "Stage $2 \mathrm{C}$ - leading edge of POP beyond the hymen."

The required sample size was based on the urodynamic findings of the FDV values before and after POP surgery from a study by Kummeling et al. ${ }^{18}$ Due to the non-normal distribution of the data, the standard deviation was calculated from the difference between the maximum and the minimum values. Using a two-tailed hypothesis test with a type 1 error of $5 \%$ and a power of $90 \%$, a minimum sample size of 116 were needed. An addition of 10 percent compensation increased the sample size to 127 participants. Statistical analysis was performed using the PASW statistics software version 18.0 (SPSS Inc., Chicago, IL, USA). Descriptive statistics were used to analyze the baseline characteristics of the patients included in the study. Continuous variables were displayed as mean \pm standard deviation if normally distributed and expressed as median (range) if not normally distributed. The McNemar test was used to compare categorical data whereas paired Student t-test was applied for continuous variables. A p-value of less than 0.05 was considered as statistical significance.

\section{Results}

A total of 291 patients underwent vaginal hysterectomy with concurrent transvaginal reconstructive procedures, except antiincontinence surgery, at the Urogynecology Unit, Siriraj Hospital during the study period. One hundred and sixty-four patients had incomplete urodynamic records. One patient was excluded due to previous history of anti-incontinence surgery, leaving a total of 126 patients for data analysis. The patients' baseline characteristics are shown in Table 1. The mean age was $69.5 \pm 8.2$ years whereas the median parity was 3 per

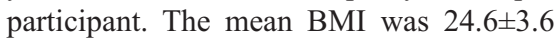
$\mathrm{kg} / \mathrm{m}^{2}$. Almost all were post-menopausal (98.4\%). Most patients (86.5\%) presented with advanced stage (stage 3 or 4 ) prolapse. Among these, advanced-stage anterior compartment prolapse was the most prevalent POP location detected (79.4\%) when com-

pared to the posterior $(22.2 \%)$ and the apical $(63.5 \%)$ compartments.

Perioperative outcomes are demonstrated in Table 2. The most frequently performed concurrent procedure was anterior vaginal mesh repair $(52.4 \%)$, followed by the obliterative total colpocleisis $(31.0 \%)$. The mean operative time for all procedures, including vaginal hysterectomy, was $85 \pm 37.8$ minutes. The only intraoperative complication encountered was massive hemorrhage (blood loss $>500 \mathrm{~mL}$ ) which occurred in 3 out of 126 patients $(2.4 \%)$ undergoing total colpocleisis.

The clinical assessment of LUTS before and after vaginal surgery for POP is illustrated in Table 3. Comparative analysis showed significant reduction in urgency (63.5\% vs 38.9\%; p < 0.001), UUI (50.8\% vs $31.7 \%$; $\mathrm{p}=0.001)$, and voiding symptoms $(42.9 \%$ vs $5.6 \%$; $\mathrm{p}<0.001)$ postoperatively. However, no significant difference was found when comparing between pre- and post-operative rates of stress and mixed urinary incontinence $(36.5 \%$ vs $34.9 \%$; $\mathrm{p}=0.88$ and $29.4 \%$ vs $20.6 \%$; $=0.09$, respectively). When further analyzed, 41 out of 80 patients $(51.2 \%)$ who previously experienced urgency reported symptom resolution after surgery. Similar findings were noted in patients presenting with UUI and voiding symptoms that 36 out of $64(56.3 \%)$ and 52 out of $54(96.3 \%)$, respectively, demonstrated remarkable improvement postoperatively. Finally, without additional anti-incontinence procedure, either therapeutic or prophylactic, persistent SUI was prevalent in $18.3 \%$ whereas de novo SUI was incident in

\section{Table 2. Perioperative outcomes.}

\section{Perioperative variables} Value $(n=126)$

Concurrent procedures

Sacrospinous fixation

Uterosacral vault suspension

Total colpocleisis

Anterior colporrhaphy

Anterior vaginal mesh repair

Operative time (min)

Complication

$\mathrm{EBL}>500 \mathrm{~mL}$

$15(11.9)$

$8(6.3)$

$39(31.0)$

$15(11.9)$

$66(52.4)$

$85 \pm 37.8$

Data presented as mean \pm Standard Deviation (SD), median (range) or number (\%) EBL: estimated blood loss.

Table 3. Pre- and post-operative LUTS.

\begin{tabular}{lccc}
\hline UUTS a & Pre-operation & Post-operation & p \\
SUI $^{b}$ & $46(36.5)$ & $44(35.0)$ & 0.88 \\
UUI $^{c}$ & $64(50.8)$ & $40(31.7)$ & $0.001^{*}$ \\
\hline MUI $^{d}$ & $37(29.4)$ & $26(20.6)$ & 0.09 \\
Urgency $^{\text {Voiding symptoms }}$ & $80(63.5)$ & $49(38.9)$ & $<0.001^{*}$ \\
\hline
\end{tabular}

Data presented as number (\%); ${ }^{*}$ statistical significance. a: LUTS: lower urinary tract symptoms; b: SUI: stress urinary incontinence; c: UUI: urgency urinary incontinence; d: MUI: mixed urinary incontinence.
$16.7 \%$ of our patients.

All patients were urodynamically evaluated at 6 months after surgery. None ceived treatment before evaluation. Comparison between pre- and post-opera-

Table 1. Patients' baseline characteristics. Baseline characteristics Value $(n=126)$

\begin{tabular}{lc} 
Age (years) & $69.5 \pm 8.2$ \\
BMI $\left(\mathrm{kg} / \mathrm{m}^{2}\right)$ & $24.6 \pm 3.6$ \\
\hline Parity & $3(0-10)$ \\
Menopause & $124(98.4)$ \\
\hline Hormone use & $2(1.6)$ \\
Preoperative POP stage & \\
Stage II & $17(13.5)$ \\
Stage III & $63(50.0)$ \\
Stage IV & $46(36.5)$ \\
\hline
\end{tabular}

Anterior

Stage $2(1.6)$

$24(19.0)$

$63(50.0)$

37 (29.4)

Posterior

$8(6.3)$

$33(26.2)$

$57(45.2)$

$26(20.6)$

$2(1.6)$

$26(20.6)$

Stage IV -

Data presented as mean \pm Standard Deviation (SD), median (range) or number (\%). 
significant differences in bladder sensation variables, including $\operatorname{FDV}(\mathrm{p}=0.518), \mathrm{SDV}$ $(p=0.385)$, and urgency or bladder capacity $(\mathrm{p}=0.287)$, were detected. When subcategorized according to the cut-off volume into increased and reduced bladder sensation, almost identical proportion of the patients from both groups were found to have increased sensation (46.0\% vs $46.8 \%$; $\mathrm{p}=1.000$ ). Only a few from both groups consistently showed reduced bladder sensation $(7.1 \%$ vs $7.9 \% ; \mathrm{p}=1.000)$. Less than $5 \%$ of the patients presented with poor bladder compliance pre- and post-operatively ( $4.8 \%$ vs $3.2 \%$; $\mathrm{p}=0.649)$. This seemed to correspond to the outcome of detrusor function that none of our patients had detrusor overactivity at baseline and only $3(2.4 \%)$ developed it after surgery. However, significant changes were observed when evaluating in terms of urethral function. Urodynamic Stress Incontinence (USI) was highly prevalent among patients having undergone vaginal surgery for POP (postoperative $31.0 \%$ vs preoperative $15.9 \%$; $\mathrm{p}=0.003$ ).

For voiding phase parameters, no statistically significant differences were identified when comparing between pre- and post-operative $\mathrm{Q}_{\max }(19.0 \pm 8$ vs $18.5 \pm 8$ $\mathrm{mL} / \mathrm{sec} ; \mathrm{p}=0.462), \mathrm{P}_{\text {det }} \mathrm{Q}_{\max }(21.0 \pm 11$ vs $\left.19.9 \pm 11 \mathrm{cmH}_{2} \mathrm{O} ; \mathrm{p}=0.179\right)$, and $\mathrm{BOO}$ (41.8\% vs 38.2\%; $\mathrm{p}=0.585$ ). Undoubtedly, reduction in PVR was apparently noted in patients who underwent surgical correction for POP (postoperative $29 \pm 41$ vs preoperative $42 \pm 54 \mathrm{~mL} ; \mathrm{p}=0.006$ ). However, twothirds of the patients significantly demonstrated weak BCI after POP repair (postoperative $61.8 \%$ vs preoperative $47.3 \%$; $\mathrm{p}=0.005$, Table 4).

Table 4. Pre- and post-operative urodynamic findings.

\begin{tabular}{|c|c|c|c|}
\hline Urodynamic findings & Pre-operation & Post-operation & p \\
\hline \multicolumn{4}{|l|}{ Filling phase parameters } \\
\hline \multicolumn{4}{|l|}{ Bladder sensation } \\
\hline $\mathrm{FDV}^{\mathrm{a}}(\mathrm{mL})$ & $158 \pm 53$ & $162 \pm 64$ & 0.518 \\
\hline $\mathrm{SDV}^{\mathrm{b}}(\mathrm{mL})$ & $263 \pm 80$ & $256 \pm 76$ & 0.385 \\
\hline Urgency (mL) & $348 \pm 103$ & $338 \pm 104$ & 0.287 \\
\hline Increased bladder sensation (FDV $<150 \mathrm{~mL}$ ) & $58(46.0)$ & $59(46.8)$ & 1.000 \\
\hline Reduced bladder sensation (SDV>250 mL) & $9(7.1)$ & $10(7.9)$ & 1.000 \\
\hline Poor bladder compliance $\left(<40 \mathrm{~mL} / \mathrm{cmH}_{2} \mathrm{O}\right)$ & $6(4.8)$ & $4(3.2)$ & 0.649 \\
\hline \multicolumn{4}{|l|}{ Detrusor function } \\
\hline $\mathrm{DO}^{\mathrm{c}}$ & $0(0)$ & $3(2.4)$ & - \\
\hline \multicolumn{4}{|l|}{ Urethral function } \\
\hline USI $^{d}$ & $20(15.9)$ & $39(31.0)$ & $0.003^{*}$ \\
\hline \multicolumn{4}{|l|}{ Voiding phase parameters } \\
\hline $\mathrm{Qmax}^{\mathrm{e}}(\mathrm{mL} / \mathrm{sec})$ & $19.0 \pm 8$ & $18.5 \pm 8$ & 0.462 \\
\hline $\operatorname{PdetQmax}{ }^{\mathrm{f}}\left(\mathrm{cmH}_{2} \mathrm{O}\right)$ & $21.0 \pm 11$ & $19.9 \pm 11$ & 0.179 \\
\hline Weak bladder contractility $(\mathrm{BCI} \mathrm{s}<100)$ & $52(47.3)$ & $68(61.8)$ & $0.005^{*}$ \\
\hline $\mathrm{PVR}^{\text {h }}(\mathrm{mL})$ & $42 \pm 54$ & $29 \pm 41$ & $0.006^{*}$ \\
\hline $\mathrm{BOO}^{\mathrm{i}}$ & $46(41.8)$ & $42(38.2)$ & 0.585 \\
\hline
\end{tabular}

[Urogynaecologia 2021; 33:264] function. Our results were incompatible with the findings from Abdullah et al. ${ }^{17}$ in which substantial increase in first desire volume, strong desire volume and bladder capacity was well established after laparoscopic sacrocolpopexy. When investigating in terms of bladder sensation parameter with regards to the cut-off volume, no significant changes, either increased or reduced bladder sensation, were observed. These reflected the outcome described by Panicker et al. ${ }^{3}$ where no difference in bladder sensation was documented after vaginal surgery for POP. Several factors might be responsible for these conflicting results. These included i) patient factors, e.g., severity of POP, co-morbidities, previous pelvic floor surgery and ii) procedure factors, e.g., route and type of surgery, surgical techniques, related complications. Moreover, age-related bladder dysfunction was probably another important factor associated with the insignificant urodynamic changes after POP repair among our patients. According to previous literatures on clinical urodynamic studies, ${ }^{25}$ advancing age has been confirmed to be correlated with reduced bladder capacity, loss of compliance, increased detrusor instability, impaired bladder contractility, decreased urine flow rate, and increased postvoid residual vol-

Our study did not find any significant changes in most of the urodynamic filling phase parameters after vaginal surgery for POP, except a significantly increased incidence of USI.

There was no significant difference note when evaluating urodynamic filling phase parameters, such as FDV, SDV, bladder capacity, bladder sensation, and detrusor

Table 5. Postoperative POP stage, POP location, and POP recurrence.

POP variables Value $(n=126)$

\section{Postoperative POP stage}

\begin{tabular}{lc} 
Stage I & $18(14.2)$ \\
Stage II & $102(81.0)$ \\
Stage III & $6(4.8)$ \\
Postoperative POP location & \\
Anterior & \\
Stage 0 & $1(0.8)$ \\
Stage I & $31(24.6)$ \\
Stage II & $88(69.8)$ \\
Stage III & $6(4.8)$ \\
Posterior & \\
Stage 0 & $16(12.7)$ \\
Stage I & $53(42.0)$ \\
Stage II & $55(43.7)$ \\
Stage III & $2(1.6)$ \\
Apical & \\
Stage 0 & $48(38.1)$ \\
Stage I & $63(50.0)$ \\
Stage II & $12(9.5)$ \\
Stage III & $3(2.4)$ \\
POP recurrence (stage) & \\
Stage II & $12(9.5)$ \\
Stage III & $6(4.8)$ \\
POP recurrence (location) & \\
Anterior & $11(8.7)$ \\
Posterior & $10(7.9)$ \\
Apical & $5(4.0)$ \\
\hline D &
\end{tabular}

Data presented as number (\%); POP: pelvic organ prolapse. 
ume. This decrement in bladder capacity probably explained why $46 \%$ of our patients were urodynamically diagnosed with increased bladder sensation (FDV < $150 \mathrm{~mL}$ ) preoperatively. Since pelvic reconstructive procedures could restore the prolapsed pelvic organs, particularly the bladder and urethra, to their usual position, it was hypothesized that the bladder could resume its normal physiology after surgery. However, our study failed to demonstrate any appreciable effect of transvaginal POP repair procedures on the bladder storage function. Hence, no significant improvement in FDV, SDV, bladder capacity, and bladder sensation was found after surgery.

Finding from this study demonstrated significantly increased incidence of USI. Nevertheless, when specifically looking at the clinical symptom of SUI, no discrepancy was found when comparing between preand post-operative outcomes. Since the number of patients with postoperative SUI derived from a combination of those having persistent symptom and those with de novo SUI, this could contribute to the comparable proportion of pre- and post-operative SUI prevalence leading to a non-significant difference between the two groups. These findings somehow reflected the failure of preoperative urodynamic test in identifying occult SUI. The explanation for this is that although prolapse reduction using a pessary could facilitate the detection of covert SUI by restoring the normal urethral anatomy, a poorly fitted or oversized pessary could possibly compress the bladder neck and urethra leading to a lower detection rate of preoperative USI. The $16.7 \%$ incidence of de novo SUI in our study was similar to that reported by Lo et al. ${ }^{14}$ (11\%) who discovered a 3.5-time greater risk of de novo SUI in women undergoing transvaginal mesh surgery. Therefore, it may be assumed that the anterior vaginal mesh repair which was performed in $52.4 \%$ of our patients was a possible predisposing factor for the occurrence of de novo SUI. In addition, the second most commonly performed reconstructive procedure, the obliterative total colpocleisis, which involved extensive dissection around the bladder neck could also contribute to the development of de novo SUI due to interference with the lower urinary tract nerve plexuses. ${ }^{14}$

Urinary urgency with or without UUI was the most prevalent LUTS manifested by two-thirds $(63.5 \%)$ of the patients preoperatively. This has proved the relationship between POP and overactive bladder (OAB). It is believed that a prominent cystocele can put traction on the urethra which results in opening of the bladder neck with urine entering the urethra subsequently inducing detrusor contractions. ${ }^{26}$ Hence, significant improvement in urgency and UUI was readily observed after surgical correction for POP due to the disappearance of bladder neck funneling as described by several research works. Remarkable improvement in voiding symptoms was also demonstrated postoperatively. This corresponded with the outcomes reported by the previously mentioned studies, ${ }^{17,27}$ suggesting that the resolution of urethrovesical angle distortion and the re-establishment of its normal anatomy could lead to symptom reduction.

As discussed earlier, the overactive bladder was associated with symptomatic POP due to prolapse-induced bladder neck funneling. Thus, surgical correction for POP could substantially improve urgency and UUI symptoms postoperatively. In addition, the effect of advancing age on bladder storage function, including loss of compliance and increased detrusor instability, undoubtedly contributed to the escalated prevalence of these OAB symptoms. Therefore, we expected to find similar outcomes when urodynamically assessing the detrusor function. To our surprise, only a few patients were proved to have poor bladder compliance and none were diagnosed with detrusor overactivity preoperatively. Perhaps, geriatric bladder dysfunction, especially impaired bladder contractility, ${ }^{25}$ was a major contributor to these inconsistent results of subjective $\mathrm{OAB}$ symptoms and objective detrusor contraction, yielding insignificant differences between pre- and post-operative urodynamic findings.

Comparative analysis regarding urodynamic voiding phase parameters exhibited a statistically significant decrease in postvoid residual volume after prolapse repair. Our result was analogous to the findings from several previous studies. ${ }^{3,16-17}$ The decline in PVR was mostly related to the beneficial effect of POP repair procedures leading to the disappearance of urethral kinking. On the contrary, no remarkable changes were observed postoperatively when evaluating in terms of $\mathrm{Q}_{\max }, \mathrm{P}_{\text {det }} \mathrm{Q}_{\max }$, and BOO. Deterioration of detrusor muscle function with subsequent impaired bladder contractility which are commonly found in elder$1 y^{25}$ could be responsible for the reduced urine flow rate, thus causing non-significant differences between the pre- and post-operative pressure-flow parameters. Moreover, prolapse reduction with vaginal pessary to accommodate urethral straightening could partly contribute to the lower incidence of bladder outflow obstruction during preoperative urodynamic assessment, resulting in inappreciable discrepancies of BOO percentage after surgery.
Weak bladder contractility was urodynamically detected among half of our patients prior to surgery. This confirmed the negative impact of geriatric changes on micturition physiology. ${ }^{25}$ When specifically looking at Schafer's BCI formula (BCI = $\mathrm{P}_{\text {det }} \mathrm{Q}_{\max }+5 \mathrm{Q}_{\max }$ ), both decreased urine flow rate $\left(\mathrm{Q}_{\max }\right)$ and impaired bladder contractility (Pdet) simultaneously accounted for the low BCI values during urodynamic study. Poor bladder contractility became even more prevalent after POP repair procedures, as evidenced by a significant increase in the number of patients having a low BCI value. Extensive dissection around the bladder neck and the vesicovaginal interface during vaginal surgery for prolapse might have caused some damage to the lower urinary tract nerve plexuses leading to defective bladder contractility postoperatively. ${ }^{28}$

\section{Strength and limitation}

Results from our study have provided analytic urodynamic assessment in patients having undergone a variety of vaginal procedures, in addition to vaginal hysterectomy. This brings about the generalizability of the data that can be applicable for a broader female population. The information obtained from this study is also useful for patient counseling regarding the choice of operation and the expected urinary outcomes after vaginal surgery for POP repair. However, this study does have some limitations. Although the surgical technique employed for vaginal hysterectomy was relatively homogeneous among the three surgeons, various concomitant vaginal procedures undertaken could yield diverse outcomes on the bladder function. Furthermore, the follow-up period for postoperative urodynamic evaluation was relatively short, as each individual received an assessment 6 months after the operation. A longer follow-up duration is needed to determine the long-term effects of vaginal reconstructive procedures on urinary symptoms and urodynamic findings. At the end of the day, a future research with a larger sample size is required to thoroughly evaluate the effect of an individual vaginal reconstructive procedure on LUTS and urodynamic parameters.

\section{Conclusions}

Transvaginal prolapse repair procedures do not have significant impact on the urodynamically assessed bladder storage function, except an increased incidence of postoperative urodynamic stress incontinence. No significant improvement in urodynami- 
cally related voiding function can be expected, except a reduction in postvoid residual urine. Finally, significant improvement in overactive and voiding symptoms can be anticipated after surgical correction for prolapse.

\section{References}

1. Haylen BT, Maher CF, Barber MD, et al. An International Urogynecological Association (IUGA) / International Continence Society (ICS) joint report on the terminology for female pelvic organ prolapse (POP). Int Urogynecol J 2016;27:165-94.

2. Jackson SL, Weber AM, Hull TL, et al. Fecal incontinence in women with urinary incontinence and pelvic organ prolapse. Obstet Gynecol 1997;89:423-7.

3. Panicker R, Srinivas S. Urodynamic Changes in Pelvic Organ Prolapse and the Role of Surgery. Med J Armed Forces India 2009;65:221-24.

4. Madhu CK, Hashim H. Surgery for pelvic organ prolapse. Eur Urol Suppl 2018;17:119-25.

5. Clark AL, Gregory T, Smith VJ, et al. Epidemiologic evaluation of reoperation for surgically treated pelvic organ prolapse and urinary incontinence. Am J Obstet Gynecol 2003;189:1261-7.

6. Brown JS, Waetjen LE, Subak LL, et al. Pelvic organ prolapse surgery in the United States, 1997. Am J Obstet Gynecol 2002;186:712-6.

7. Lensen EJM, Withagen MIJ, Kluivers $\mathrm{KB}$, et al. Urinary incontinence after surgery for pelvic organ prolapse. Neurourol Urodyn 2013;32:455-9.

8. Ugianskiene A, Kjargaard N, Lindquist ASI, et al. Retrospective study on de novo postoperative urinary incontinence after pelvic organ prolapse surgery. Eur J Obstet Gynecol Reprod Biol 2017;219:10-4.

9. Frigerio M, Manodoro S, Palmieri S, et al. Risk factors for stress urinary incontinence after native-tissue vaginal repair of pelvic organ prolapse. Int J Gynecol Obstet 2018;141:349-53.

10. Alas AN, Chinthakanan O, Espaillat L, et al. De novo stress urinary incontinence after pelvic organ prolapse surgery in women without occult incontinence. Int Urogynecol J 2017;28:58390

11. Long CY, Hsu SC, Wu TP, et al. Urodynamic comparison of continent and incontinent women with severe uterovaginal prolapse. J Reprod Med 2004;49:33-7.

12. Richardson D, Bent A, Ostergard D. The effect of uterovaginal prolapse on urethrovesical pressure dynamics. Am J Obstet Gynecol 1983;146:901-5.

13. Bump RC, Fantl JA, Hurt WG. The mechanism of urinary continence in women with severe uterovaginal prolapse: results of barrier studies. Obstet Gynecol 1988;72:291-5.

14. Lo TS, Bt Karim N, Nawawi EA, et al. Predictors for de novo stress urinary incontinence following extensive pelvic reconstructive surgery. Int Urogynecol J 2015;26:1313-9.

15. Foster Sr RT, Barber MD, Parasio MF, et al. A prospective assessment of overactive bladder symptoms in a cohort of elderly women who underwent transvaginal surgery for advanced pelvic organ prolapse. Am J Obstet Gynecol 2007;197:82.e1-4.

16. Illiano E, Natale F, Giannantoni A, et al. Urodynamic findings and functional outcomes after laparoscopic sacrocolpopexy for symptomatic pelvic organ prolapse. Int Urogynecol J 2019;30:589-94.

17. Abdullah B, Nomura J, Moriyama S, et al. Clinical and urodynamic assessment in patients with pelvic organ prolapse before and after laparoscopic sacrocolpopexy. Int Urogynecol J 2017;28:1543-9.

18. Kummeling MTM, Rietbergen JBW,
Withagen MIJ, et al. Sequential urodynamic assessment before and after laparoscopic sacrocolpopexy. Acta Obstet Gynecol Scand 2013;92:172-7.

19. Haylen BT, de Ridder D, Freeman RM, et al. An International Urogynecological Association (IUGA)/International Continence Society (ICS) joint report on the terminology for female pelvic floor dysfunction. Neurourol Urodyn 2010;29:4-20.

20. Drake MJ, Doumouchtsis SK, Hashim $\mathrm{H}$, et al. Fundamentals of urodynamic practice, based on International Continence Society good urodynamic practices recommendations. Neurourol Urodyn 2018;37:S50-60.

21. Yao M, Simoes A. Urodynamic Testing and Interpretation. [Updated 2020 Sep 1]. In: StatPearls [Internet]. Treasure Island (FL): StatPearls Publishing; 2021 Jan-. Available from: https://www.ncbi.nlm.nih.gov/books/N BK562310/

22. Blaivas JG, Groutz A. Bladder outlet obstruction nomogram for women with lower urinary tract symptomatology. Neurourol Urodyn 2000;19:553-64.

23. Nitti VW. Pressure flow urodynamic studies: the gold standard for diagnosing bladder outlet obstruction. Rev Urol 2005;7:S14-21.

24. Barber MD, Brubaker L, Nygaard I, et al. Defining success after surgery for pelvic organ prolapse. Obstet Gynecol 2009;114:600-9.

25. Siroky MB. The aging bladder. Rev Urol 2004;6:S3-7.

26. Boer TA, Salvatore S, Cardozo L, et al. Pelvic organ prolapse and overactive bladder. Neurourol Urodyn 2010;29:30-9.

27. Romanzi LJ, Chaikin DC, Blaivas JG. The effect of genital prolapse on voiding. J Urol 1999;161:581-6.

28. Miyazato M, Yoshimura N, Chancellor MB. The other bladder syndrome: underactive bladder. Rev Urol $2013 ; 15: 11-22$. 